\title{
Personalized Medicine: connecting the dots in a bonanza of science and technology
}

Soulla Louca ${ }^{1}$

1 Management \& MIS, Faculty Member, University of Nicosia

Our society is challenged with several health care issues ranging from an aging population where health care is becoming too expensive to chronic diseases becoming far more prevalent. Improved disease diagnosis for more effective and precise disease treatments should be obligatory. Personalized Medicine (PM) representing a more defined and operative patient-centered care and aims to provide the answer to a health care system in which patient care is consistently improved through the use of information on the individual patient's genomes and their downstream implications. PM is a rapidly emerging area aspiring to revolutionize the health care system by providing significant benefits for patients and a large economic impact across public and private sectors. It promises citizens more effective, safer and cheaper treatments, genetic prediction prevention of disease, and empowerment through participatory medicine. The pharmaceutical industry is looking at new opportunities, and new markets for new drugs, opening at the same time the diagnostic and medical devices fields. At the same time the information, communication technology (ICT) industry is investigating the potential for developing new testing models and reimbursement bodies are looking forward to reduced health care expenditure. The time to start identifying and connecting the dots in PM is now. Realizing its potential requires new methods for processing the deluge of genomic data and translation of the findings into medical practice and the development of affordable, next generation, high-throughput technologies for the generation of data from the entire genome, transcriptome, epigenome, etc, from a single (routine) clinical specimen. While challenges still exist, the capturing of biology in software and hardware through the modeling of genes, proteins, cells, and human organs along with data gathering, simulation, and visualization, ICT inevitably becomes the critical enabler of PM. This upheaval in the life sciences requires new computing capabilities, sophisticated algorithms, a vast range of software products, internet technologies, as well as advanced data management capabilities and standardization protocols facilitating simultaneous evolvement of ICT. As a result, the exploration of strategic relationships among various disciplines such as life sciences, mathematics, physics, chemistry, and ICT, and constellation thinking to propose new ways for the diagnosis and therapy of diseases, integrated with a planned trans-disciplinary scientific approach involving all interested parties, is required. 
Increased training and education for scientists, medical practitioners, entrepreneurs and other personnel for designing, using and maintaining these new environments, which employ a whole new approach for the drug development process is necessary as well. Preparing physicians for systems thinking and integrated team approaches will require significant changes in education and training for new and established physicians. The education of the public as a whole and the societal engagement on these novel developments remains a difficult challenge. Issues regarding patients as well as caregivers involvement in research need to be addressed. An informed patient is a cost effective patient who deserves the best treatment possible, including readily and quickly available information on new treatment opportunities. Health literacy will not only encourage patients and the public in general to be more uttered about their needs, but it will also assist to 'pull' PM from the ground up.

Patient empowerment with predictive and preemptive information to manage their own health by being able to harness the knowledge to keep on being healthy or take advantage of treatments tailored to their genetic profile will also enable individuals to know ahead of time whether they are susceptible to certain diseases. Several social and ethical issues need to be addressed. For example, what if parents decide at an early age that they want to know more about the genetic profile of their child but, as an adult, that child might not want to know what awaits him/her in the future? There is an urgent need to identify what we actually want to predict and where to intervene since a wrong prognosis/interpretation will generate serious problems that will follow a child for the rest of his/her life. In addition, an ideal health care system is one that is accessible to everybody when clinically beneficial new products and procedures are translated into affordable clinical practice. Access to this foretelling information should not be the privilege of the few. Personalized Medicine is data-rich. Questions relating to the quality and validation, integration, interpretation, application and confidentiality of personal data (especially genetic data) along with ensuring benefits for patients and society from its use in clinical practice and research purposes arise. Data sharing and equal access to tools remain difficult issues to address. Policy challenges, including encouraging and financing innovation related to the field, need to be surmounted for effective individually tailored medical interventions, simultaneously reforming the health care system. A global harmonized and standardized ethical and regulatory system that will take into consideration reimbursements, data standards, large infrastructures and open systems, the intellectual property landscape, laws or regulations for nanomedicine is required.

Rules and regulations for approval of clinical studies for this emerging paradigm of targeted drugs which impact understood biological pathways need to be modified. Encouraging investment in better targeted medicine and diagnostics needs to be enhanced. Efficient use of healthcare budgets and the availability of venture capital healthcare costs for an improved research structure and availability of resources and equipment for new drug developments, diagnostics and pre-emptive testing, training and so on is a must. Diseases are being redefined and reclassified, the patient is ready to participate, and regulators and policy makers are initiating actions. Most of the tools and components, namely the science, the technology, the social, ethical and legal issues, the education and training, the public (payers and patients), the reimbursement agencies, the industry (pharmaceutical and technological), the policy makers and the clinicians, required for the implementation of PM are in place. What remains to be identified are the various links between them. The key actors in linking these challenges are the citizens. They are the drivers of a cultural change in which the right to control individual data enshrines to a duty to use it judiciously and develops into proactive actions for managing their own health for participatory medicine. The foundations for a new health care system mounting the opportunity to turn this overwhelming bonanza of science and technology into personalized medicine have been laid. Now is the time to build upon them.

'It is not the strongest of the species that survives nor the most intelligent that survives. It is the one that is most adaptable to change.' Charles Darwin, 1809 - 1882 